(c) American Dairy Science Association, 2006.

\title{
Spatial and Temporal Patterns of Herd Somatic Cell Score in France
}

\author{
E. Gay, ${ }^{*} \dagger$ J. Barnouin, $\dagger^{1}$ and R. Senoussi ${ }^{*}$ \\ *Unité de Biométrie, INRA Domaine St-Paul, Site Agroparc, 84914 Avignon Cedex 9, France \\ †Unité d'Epidémiologie Animale, INRA, 63122 St-Genès-Champanelle, France
}

\section{ABSTRACT}

Spatial and temporal patterns of annual milk somatic cell score (ASCS) were explored in French dairy herds between 1996 and 2000 to detect regional singularities for risk of mastitis. A new cluster detection method was used, which was adapted to continuous variables and which allowed ASCS variation factors to be taken into account. The statistical unit was the herd-year. A linear regression model for each year allowed adjustment for breed, mean parity, number of calvings for each season, herd size, and farm altitude. Cluster detection was performed on raw data and on residuals of the model through a method based on the Hellinger distance between spatial distributions. The Hellinger distance between farm distributions was computed at different levels of ASCS (or residuals). Temporal ASCS patterns were explored using a computation of correlations and comparisons between spatial structures of the different years. The general ASCS trend over the study period was a decrease. The global Hellinger distance, which was higher than what could have been randomly expected for each of the 5 yr, indicated a significant spatial cluster formation. Cluster mapping over the $5 \mathrm{yr}$ identified several areas, which sometimes differed between detection using raw data and that using ASCS residuals. Temporal correlations between ASCS residuals for each year were positive and decreasing, and 1996 and 2000 appeared spatially different from the other years. The more affected areas were regions that were not specialized in dairy production. During the study period, 2 progressive movements were detected, corresponding to a disappearance of clusters in the northwest and an increase of clusters in the southwest. Cluster detection could aid in the identification of new risk factors that are relevant at different spatial scales, and could help local organizations to supervise the risk of mastitis, and improve udder health management.

Key words: spatial and temporal pattern, somatic cell score

Received September 13, 2005.

Accepted February 15, 2006

${ }^{1}$ Corresponding author: barnouin@clermont.inra.fr

\section{INTRODUCTION}

The udder health status of the dairy cow is reliably approached by the determination of milk SCC, because the main factor causing an increase above the SCC physiological level is inflammation caused by infection (Harmon, 1994). The log-transformed SCC, or somatic cell score $\left[\mathrm{SCS}=\log _{2}(\mathrm{SCC} / 100,000)+3\right]$, is an internationally recognized indicator for mastitis and milk quality (Ali and Shook, 1980). This transformation allows for normal distribution of the SCS. Moreover, cow SCC are available in many countries on a monthly basis through the DHIA.

Risk and protection factors associated with SCS have been widely investigated in observational studies (Barkema et al., 1998; Busato et al., 2000; Barnouin et al., 2004) that have highlighted the influence of cow characteristics (breed, parity), dairy management practices (milking, udder hygiene, housing system, calving conditions), seasonal factors (climate), and interactions among these different factors on the SCS level.

Moreover, differences in natural resources, dairy farm structures, and market conditions cause different regions of the same country to implement different dairy management systems, which may be reflected in various levels of technical performance (Oleggini et al., 2001; Ely et al., 2003). Consequently, the assessment of regional differences in SCS through the study of spatial and temporal patterns can assist the DHIA in formulating regional programs focused on local factors of milk quality improvement. In the United States, Ely et al. (2003) explored spatial variations of some key variables in dairy management, including SCS, and the differences between regions were noticeable.

Numerous studies have focused on the use of methods for identifying and analyzing spatial or temporal clusters of animal disease events (Ward and Carpenter, 2000; Carpenter, 2001). However, these events are essentially concerned with dichotomous variables (e.g., cases and controls), and not with continuous variables such as SCS.

To explore spatiotemporal clustering, it is first necessary to specify a cluster definition (Wartenberg, 2001). A cluster could be defined as a geographically 
and temporally bound group of close values that is unlikely to have occurred at random (Elliott and Wakefield, 2001). The interest very often focuses on unexplained clustering (Marshall, 1991), but the methods used rarely take into account known disease risk factors. Nevertheless, a way to deal with this problem is to statistically model the disease and then use the results to perform the cluster detection (Timander and McLafferty, 1998; Ahrens et al., 2001).

Once the cluster definition is specified, a method must be chosen to detect clusters. Scan statistics are widely used to track disease events (Norstrom et al., 1999; Perez et al., 2002). These methods compare the observed number of events inside a moving window to the expected number of events under a Poisson or a Bernoulli hypothesis. Yet such approaches, which deal only with binary variables, are not suitable for the detection of clusters among SCS.

The aim of this paper is to analyze the spatial and temporal patterns of SCS in France. To achieve this aim, we used an original cluster detection approach adapted to continuous variables. The method was based on the Hellinger distance between spatial distributions, and took into account known SCS variation factors.

\section{MATERIALS AND METHODS}

\section{Data}

The study population consisted of a cohort of French dairy herds enrolled in DHIA during the period from 1996 to 2000 (52,000 herds). The selected herds were pure breeds (100\% of cows of the same breed) and had at least 20 cows. Following these criteria, the eligible herds numbered 34,735 . As a compromise between the necessary representativeness of the sample and the computational facilities needed for a new approach, we selected a uniform random sample representing $15 \%$ of the eligible herds (10\% of the national dairy herds) in the national DHIA database (i.e., 5,210 farms).

Data concerning the disease were extracted from the national DHIA database containing monthly data. A standard method based on fluoro-opto-electronic cell counting (Schmidt-Madsen, 1975) was used to measure individual SCC. The outcome variable was the annual herd SCS (ASCS), which was computed as the arithmetic mean of all monthly cow SCS values determined in a herd during a calendar year. The explanatory variables extracted from the DHIA database were breed, mean parity, number of calvings per season, and herd size. No other SCS variation factor was available for the sample of herds selected from the DHIA database. Annual means or counts were calculated from monthly data. Calving seasons were classified according to Huffman et al. (1984) as spring (March to May), summer (June to August), autumn (September to November), and winter (December to February). The farm geographic coordinates (farmer address) and altitude were obtained via the French National Institute of Statistics and Economic Studies. The final database built contained 1 line per herd per year (i.e., a herd-year statistical unit).

\section{Statistical Analysis}

Statistical procedures were conducted using R 2.0.1 ( $R$ Development Core Team, 2004). The specific programs implemented to analyze our data set are available on request.

After a descriptive analysis of the study variables, we used mapping representations to explore spatial and temporal patterns of the data. When a spatial interpolation was needed to represent local farm density and ASCS intensity, we used a kernel smoothing technique, choosing the parameters recommended by Silverman (1986).

As a second analysis step, we performed a linear regression model of ASCS for each year, to take into account the available risk factors (breed; mean parity; number of calvings in the spring, summer, autumn, and winter; herd size; and farm altitude). Linear regression is the basic statistical method used to study the effects of risk factors on SCS (Laevens et al., 1997; Barkema et al., 1999). Correlations between covariables were computed before modeling to ensure that the factors included were independent. Variables were included in the model as follows:

- 3 dummy variables for breed: We chose Holstein as the reference group and introduced 3 other groups into the model (i.e., the Montbéliarde, the Normande, and "other breeds");

- 1 variable for mean parity;

- 4 variables for number of calvings occurring in the spring, summer, autumn, and winter, respectively;

- 1 variable for herd size (10 cows per unit); and

- 1 variable for farm altitude (10 m per unit).

The model assumptions were assessed through a Kolmogorov-Smirnov test of the Gaussianity of the residuals and by using a plot of residuals vs. predicted values to check homoscedasticity (Draper and Smith, 1996). The residuals could be considered as the unexplained part of the ASCS, following removal of the effects of the integrated risk factors and the mean value. 
In the third analysis step, the aim was to test whether the ASCS (or the residuals) were spatially randomly distributed or whether there were localized foci of high values. The analysis was performed on both the raw data (ASCS) and the residuals of the regression model.

The heterogeneity of the spatial distribution of farms generally leads to a poor estimation of farm density, and so makes it difficult to visualize the foci: Many high values would be likely if there were many farms in the area (Waller, 2000). To overcome this difficulty, we used a homogenization technique of point distribution before calculation via a radial deformation of the space. The deformation allowed local dilation or contraction of the space in accordance with the number of points, without changing the neighborhood (Senoussi et al., 2000). Consequently, the farm pattern became homogeneous with a nearly constant density.

For any given ASCS level $z_{j}$, we then compared the normalized density $p_{z_{j}}$ of the $n_{j}$ farm locations $x_{i}$ whose ASCS values were $e_{i} \geq z_{j}$ to the initial farm density $p_{z_{0}}$ via the Hellinger distance in the spatial domain $D$ (Gibbs and $\mathrm{Su}, 2002$ ):

$$
\operatorname{Hel}\left(z_{j}\right)=\sqrt{\frac{1}{2} \int_{D}\left(\sqrt{\bar{p}_{z_{0}}(x)}-\sqrt{\overline{\mathrm{p}}_{z_{j}}(x)}\right)^{2} d x .}
$$

The integral over domain $D$ was approximated by calculation on a regular grid. This formula gave a Hellinger distance value for each level $z_{j}$. Having chosen the number of levels dividing the range of ASCS/residuals, we defined the global Hellinger distance $(\mathrm{Hel})$ as the sum of those different values weighted by the farm count at each level ( $j$ levels):

$$
H e l=\sum_{j} \operatorname{Hel}\left(z_{j}\right) \frac{n_{j-1}-n_{j}}{n} .
$$

Under the null hypothesis $\mathrm{H}_{0}$, "the values are independently distributed in space," the farm distribution at any level should be close to the initial farm distribution, and the Hellinger distance should therefore be low. To test this hypothesis of complete spatial randomness, we used spatial permutations. The permutation procedure randomly assigns the existing values (here, the ASCS or residuals for a specified year) over the geographical points (Manly, 1991). All possible permutation results yielded the randomization distribution. We calculated the symmetric quantile interval supporting 95\% of the global Hellinger distance under the null hypothesis of no spatial structure of the data (1,000 permutations, bilateral risk, $\alpha=5 \%)$. A high value of the observed global Hellinger distance outside this quantile interval, carried out by the permutations, allowed the null hypothesis to be rejected. Consequently, the values were considered to be spatially structured, and some areas presented clusters whereas others were emptier than expected by random.

Following this global testing, the Hellinger distance was mapped at the different levels to identify significant levels and areas. To select the farms involved in areas of concentration and report them on the original map, we chose a level at which the clusters were clearly defined and where the point number was sufficient for calculation (at least $10 \%$ of the total number of points).

In a last analysis step, we explored the temporal stability of spatial patterns by 2 complementary approaches. The first one was descriptive and computed the temporal correlations between residuals of each year. The second approach was inferential and used temporal permutation techniques: A value was randomly assigned to each point among the 5 possible ASCS residuals for the 5 yr (1,000 permutation samples, independent permutations for each farm), and the global Hellinger distance was calculated. This allowed us to test the temporal discrepancy of a particular year compared with the entire 5-yr period.

\section{RESULTS}

\section{Descriptive Analysis}

Eighty-one percent of herds had Holstein cows, whereas 13\% had Montbéliarde cows, $5 \%$ had Normande cows, and $1 \%$ had "other breeds." The mean farm altitude was $226 \mathrm{~m}(\mathrm{SD}=244)$. According to the year, ASCS varied from 3.05 to 3.17 , which corresponded to 253,000 to 262,000 cells $/ \mathrm{mL}$, and it decreased over the final $2 \mathrm{yr}$ (1999 and 2000; Table 1). Mean parity decreased progressively during the study period. The other farm characteristics were stable over the 5-yr period. The geographic distribution of the farms is illustrated in Figure 1, where (a) represents the location of the selected farms, and (b) is the density of the selected farms obtained by a kernel smoothing technique. The kernel parameters used were a Gaussian function and a bandwidth parameter of 0.014. The farm density was geographically nonhomogeneous, and 3 areas had higher densities: 1) the northwest, which is the main dairy area in France; 2) the north; and 3) the east-central area. By contrast, the southeast (i.e., the Mediterranean area) had very few dairy farms.

The maps of ASCS spatial distribution (Figure 2) showed that during the entire study period, the northcentral area had relatively high ASCS intensities of 
Table 1. Characteristics of the sample of dairy herds in France during the study period ( $\mathrm{n}=5,210,1996$ to 2000)

\begin{tabular}{|c|c|c|c|c|c|}
\hline Characteristic & 1996 & 1997 & 1998 & 1999 & 2000 \\
\hline Annual SCS & $3.12(0.59)^{1}$ & $3.17(0.58)$ & $3.14(0.57)$ & $3.07(0.55)$ & $3.05(0.57)$ \\
\hline Herd size & $40.2(16.7)$ & 40.5 (16.7) & $39.9(16.6)$ & $40.1(16.7)$ & $39.9(16.6)$ \\
\hline Mean parity & $2.62(0.33)$ & $2.61(0.33)$ & $2.56(0.32)$ & $2.49(0.32)$ & $2.46(0.31)$ \\
\hline \multicolumn{6}{|l|}{ Calving, \% } \\
\hline Spring & 13.1 & 12.9 & 13.3 & 12.9 & 13.6 \\
\hline Summer & 21.9 & 21.7 & 21.3 & 21.6 & 22.6 \\
\hline Autumn & 40.9 & 41.2 & 41.2 & 40.8 & 39.6 \\
\hline Winter & 24.1 & 24.2 & 24.2 & 24.7 & 24.2 \\
\hline
\end{tabular}

${ }^{1}$ Mean (standard deviation).

around 3.5 to 4 . Nevertheless, during the last $2 \mathrm{yr}$, this area was notably reduced. Some spots with values higher than the surrounding areas appeared from 1998 in the southwest. The high value observed in the south, at the border of the Mediterranean area, was due to an isolated, high-valued point, as seen in Figure 1a (the second southern-most point in Figure 1a). This appeared to be an artifact of the general smoothing technique and could be corrected via a homogenization method.

The map of farms experiencing 4 successive ASCS increases or decreases is presented in Figure 3. A total of 106 farms had 4 consecutive increases $(2.0 \%$ of the farms), whereas 200 experienced 4 consecutive decreases (3.8\% of the farms). As already seen with the ASCS in Table 1, the general trend was a decrease, which was lower in the last $2 \mathrm{yr}$. But the concentration of farms with 4 decreasing ASCS was higher in the northwest and along the border of the east-central area. By contrast, numerous farms located in the southwest had 4 successive increases, whereas no farms showed 4 successive decreases.

\section{Regression Model}

All ASCS regression models were significant (Table 2 ), and the $R^{2}$ varied between 10 and $15 \%$. Moreover, the hypothesis of normality of residuals was not rejected by the Kolmogorov-Smirnov test for any year, and the plot of residuals vs. predicted values did not reject the hypothesis of homoscedasticity.

Finally, according to the regression models, ASCS was significantly raised with increased mean parity and increased numbers of spring calvings. By contrast,
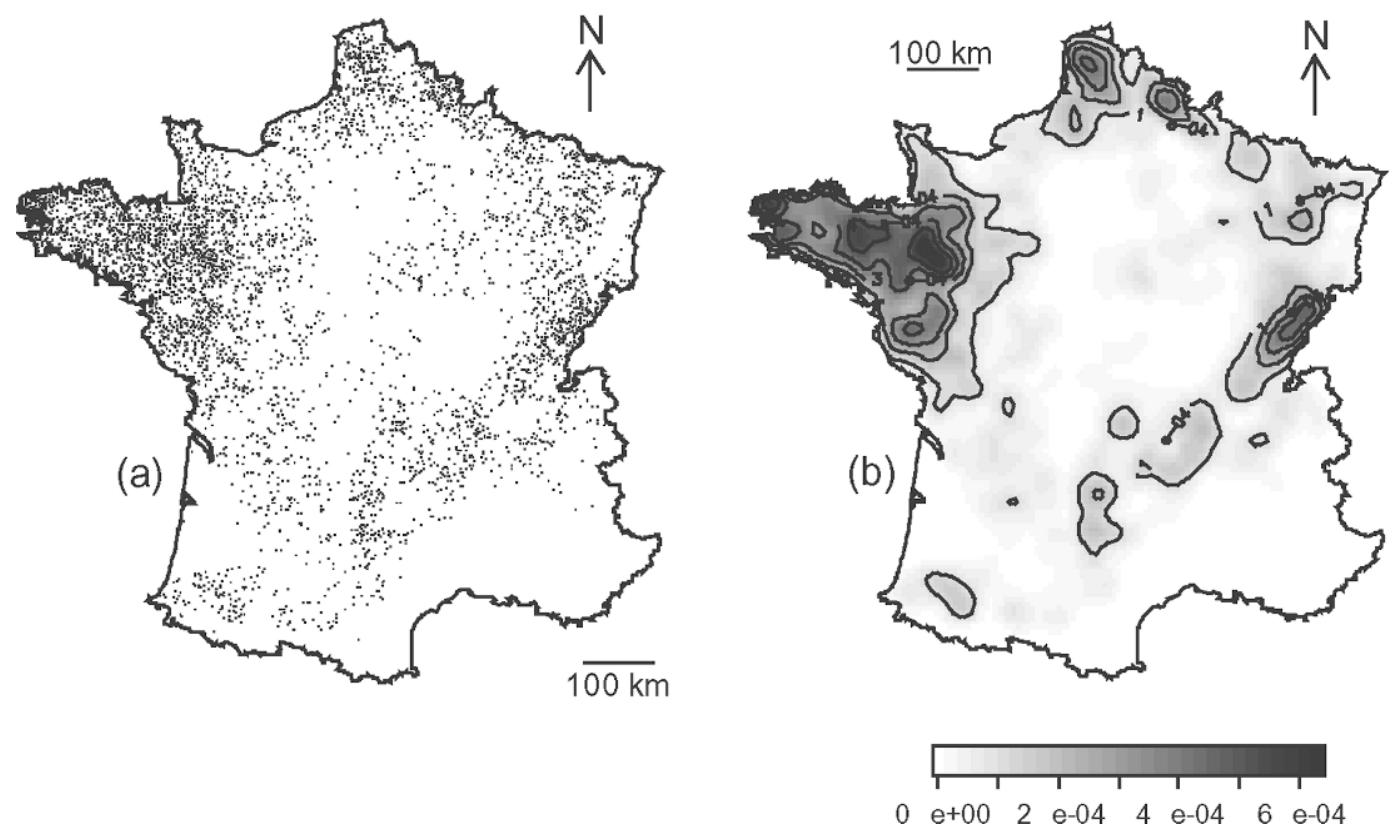

Figure 1. Spatial distribution of the study sample (dairy herds in France, $\mathrm{n}=5,210$ ): (a) farm locations and (b) farm density (farms $/ \mathrm{km}^{2}$ ). 

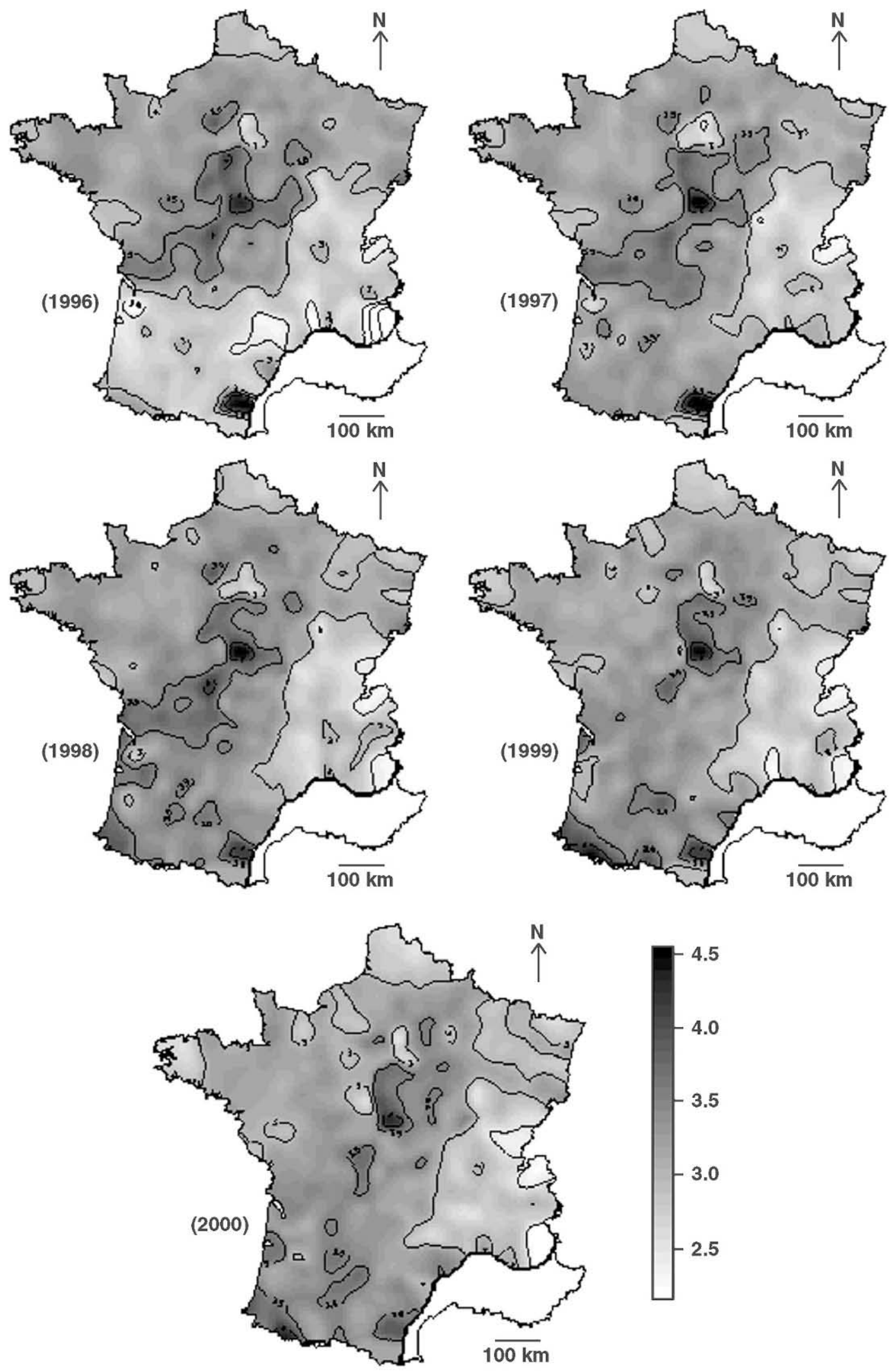

Figure 2. Annual SCS intensity in the study sample (dairy farms in France, $n=5,210,1996$ to 2000). 


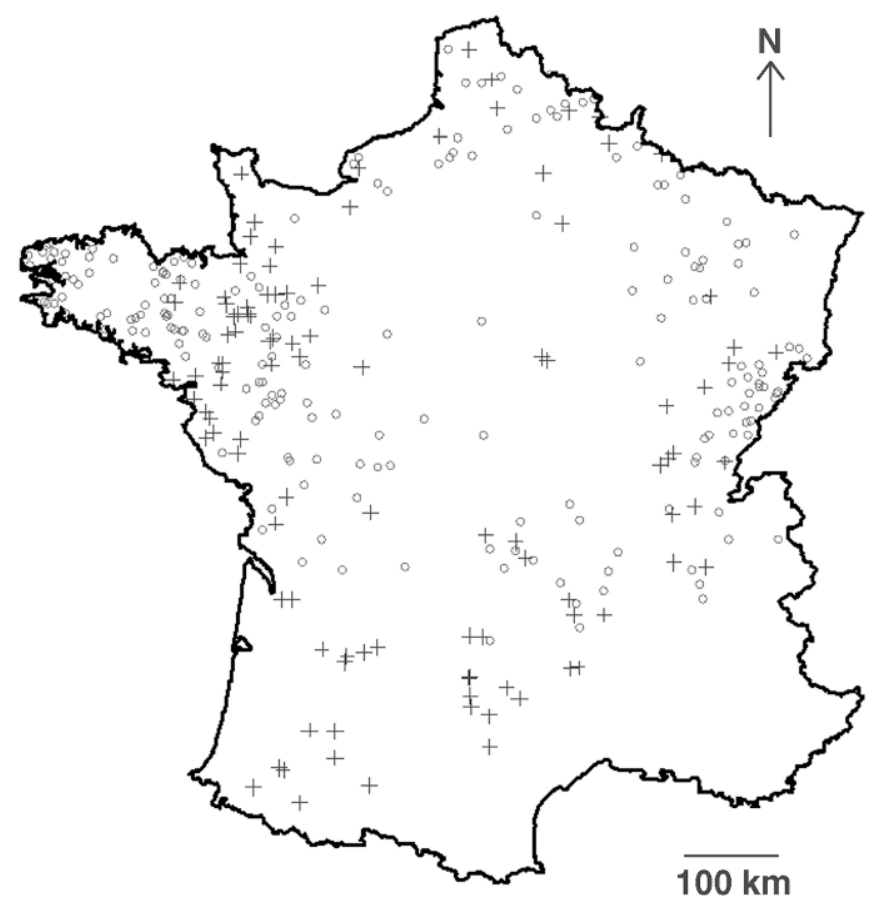

Figure 3. Dairy farms included in the 5 -yr period with 4 successive annual SCS increases or decreases. (+) 4 successive increases; (○) 4 successive decreases.
ASCS was significantly decreased by an increased number of calvings in the summer (except in 1996) and autumn (except in 2000) and by an increased altitude. For each year studied, the breed factor was highly significant, and the influence of each breed compared with the Holstein taken as a reference was as follows: the Montbéliarde decreased ASCS, and the Normande increased the score. The herd size had no significant influence, except in 1997 when an increased herd size increased ASCS.

\section{Cluster Detection}

The spatial deformation of farm distribution is displayed in Figure 4. The map of homogenized farm density was used as the reference map to visualize further concentration with increasing values of ASCS or residuals.

Detection Using Raw Data (ASCS). The observed global Hellinger distance statistic and the symmetric quantile interval of the randomization distribution are presented in Table 3. Because the global Hellinger distance value was beyond the bounds of $\mathrm{H}_{0}$ for the 5 yr studied, the hypothesis of an absence of spatial cluster formation of the ASCS was rejected.

The visualization of herds involved in the clusters was performed at level 3.8 (the level at which the clus-

Table 2. Linear regression model of the annual SCS in a sample of dairy herds in France (n = 5,210, 1996 to 2000)

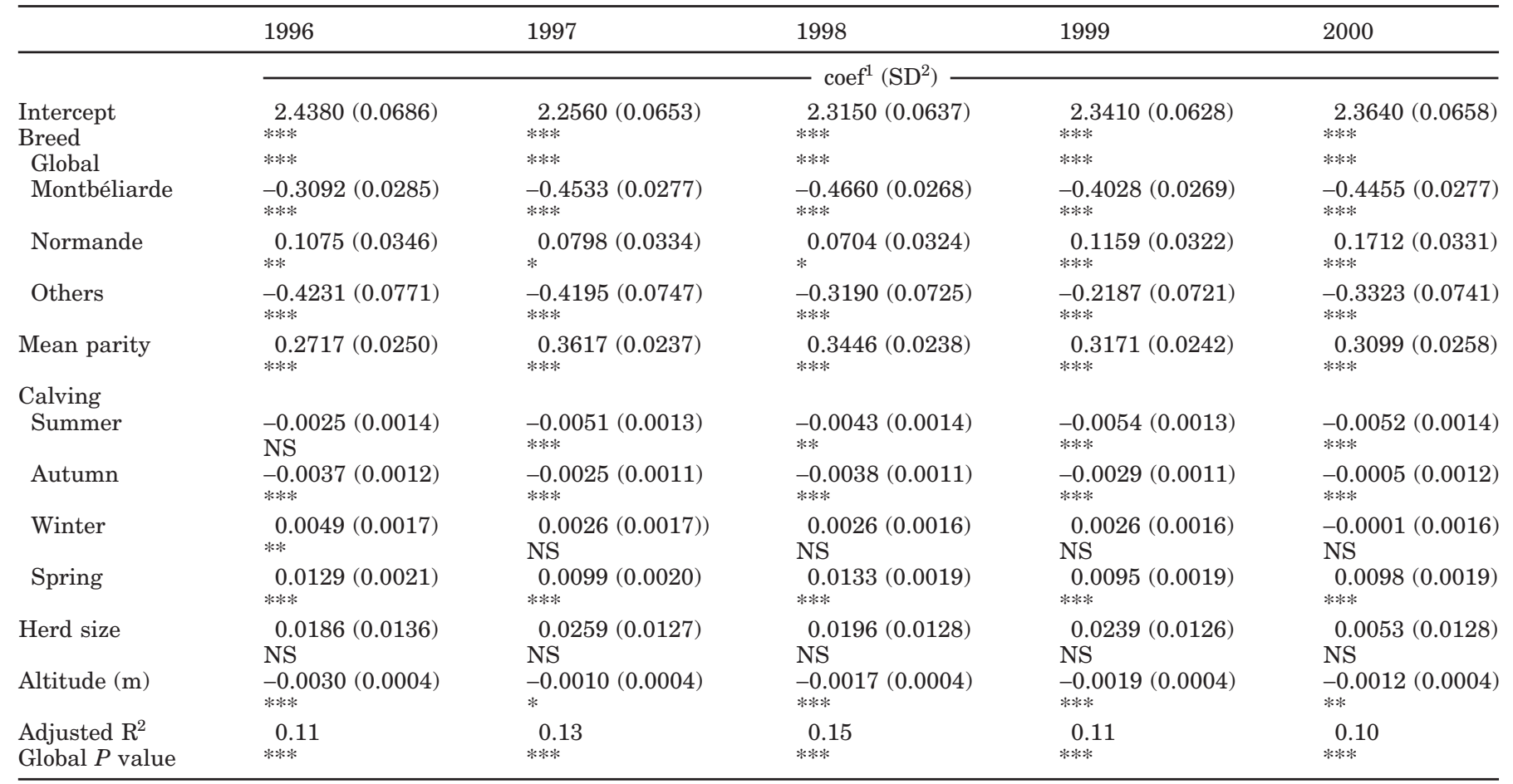

$* P<0.05 ; * * P<0.01 ; * * * P<0.001 ; \mathrm{NS}=P \geq 0.05$.

${ }^{1}$ Coefficient estimation.

${ }^{2}$ Standard deviation. 

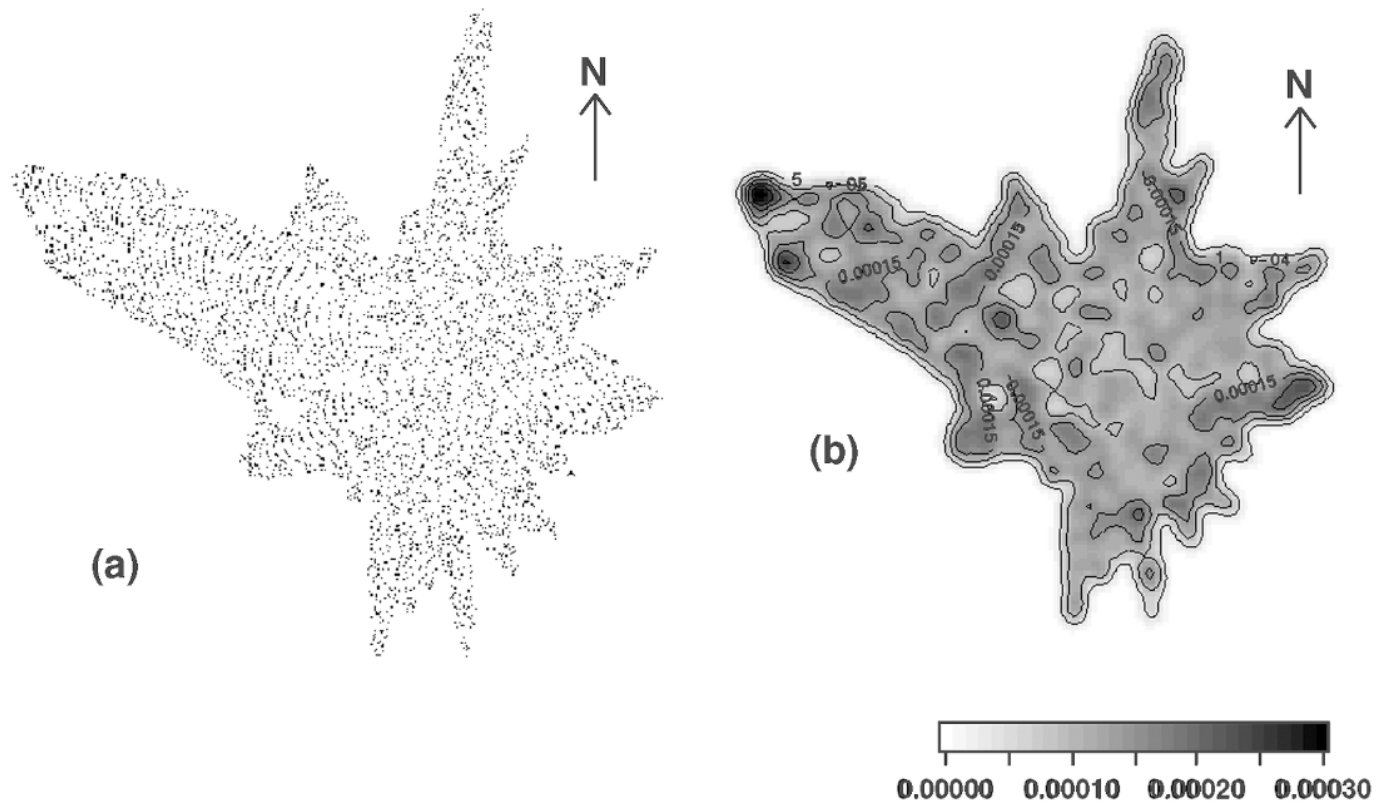

Figure 4. Spatial distribution after deformation (dairy herds in France, $\mathrm{n}=5,210$ ): (a) farms and (b) farm density $\left(\right.$ farms $/ \mathrm{km}^{2}$ ).

ters were visually clearly defined and where the point number was sufficient for calculation), and is presented in Figure 5. Figure 6 shows the summary of the cluster detection results for the $5 \mathrm{yr}$. In northwestern France, 3 areas were detected: 2 around the cities of St-Brieuc and Vannes from 1996 to 1998, and one around the city of St-Lo in 2000. An area around Auxerre in north-central France was detected in 1997 and 1999. In the southwest, a cluster was detected for each of the $5 \mathrm{yr}$ and spread progressively southward with increasing years: From the city of Limoges in 1996 and 1997, it spatially ended near the city of Agen in 1998 and reached Toulouse in 1999 and 2000.

Detection Using ASCS Residuals. The observed global Hellinger distance statistic and the symmetric quantile interval of the randomization distribution are presented in Table 4. Again, because the global Hellinger distance value was beyond the bounds of $\mathrm{H}_{0}$ for any year, the hypothesis of absence of spatial cluster formation of the ASCS residuals for the years 1996 to 2000 was rejected.
The visualization of herds involved in the clusters was performed at a residuals level of 0.64 . Figure 7 shows a summary of cluster detection results for the 5 yr. In northwestern France, 4 areas were detected: around the city of St-Brieuc in 1996 and 1997; around the city of Vannes from 1996 to 1998; and 2 areas around the city of Angers in 1997, 1998, and 2000. The area around Auxerre in north-central France persisted over the $5 \mathrm{yr}$. In the southwest, we detected a similar movement as for the raw data (i.e., a cluster progressively spreading to the south). Several other clusters appeared in northeastern France, around the city of Charleville-Mézières in 1997 and 2000, close to the city of Strasbourg in 1966, and around Vesoul and Bourg-en-Bresse in 2000. A final cluster was detected around the northern city of Amiens.

Temporal Patterns. Temporal correlations between ASCS residuals for each year were positive and decreasing: At the 1-yr lag (1996 to 1997, 1997 to 1998, 1998 to 1999,1999 to 2000 ) the correlation was 0.76 , whereas it was 0.64 at the 2 -yr lag (1996 to 1998,1997

Table 3. Global Hellinger distance and quantile interval of the randomization distribution for the analysis of annual SCS (dairy herds in France, $\mathrm{n}=5,210,1996$ to 2000$)$

\begin{tabular}{llllll}
\hline & 1996 & 1997 & 1998 & 1999 & 2000 \\
\hline $\begin{array}{l}\text { Global Hellinger distance } \\
\begin{array}{l}\text { Quantile interval of the } \\
\text { randomization distribution } \\
\text { (bilateral risk of } 2.5 \%)\end{array}\end{array}$ & 0.1726 & 0.1778 & 0.1823 & 0.1705 & 0.1796 \\
\hline
\end{tabular}




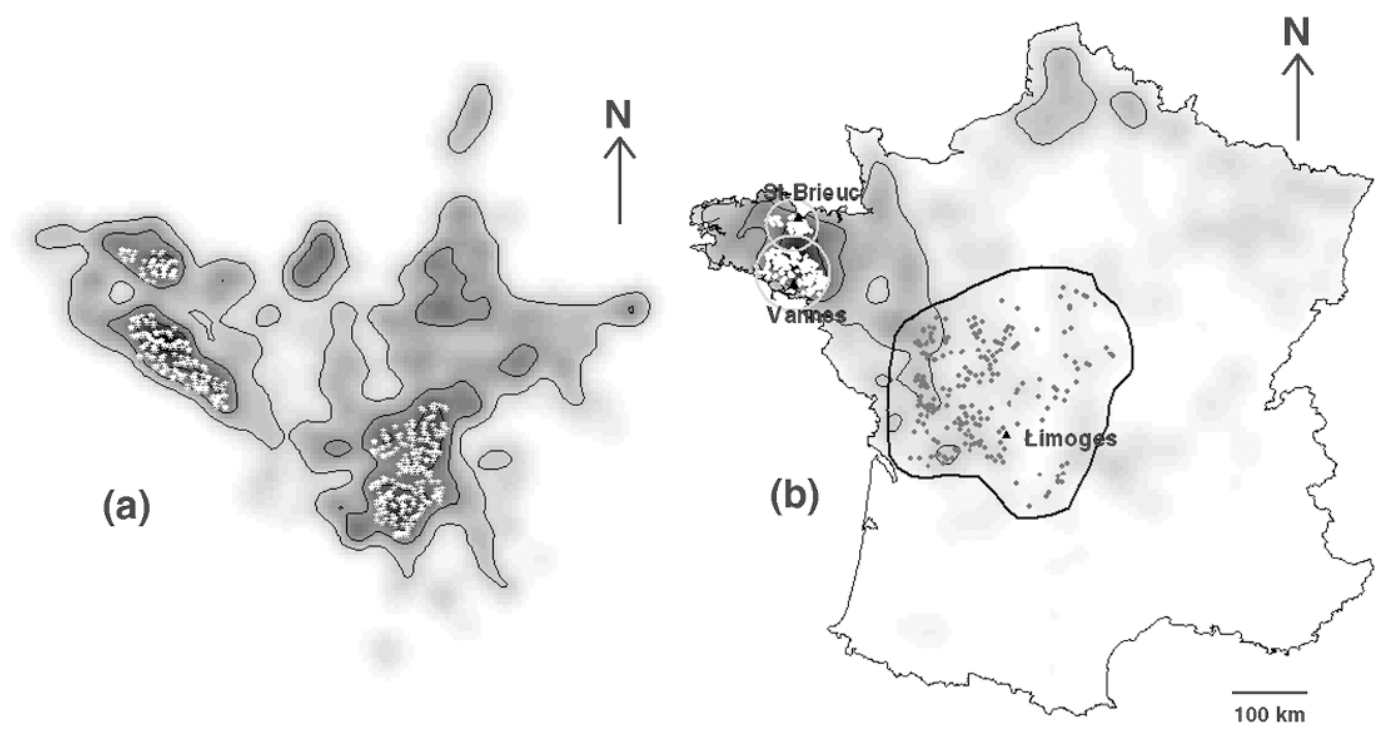

Figure 5. Localization of clusters of annual SCS in 1996: (a) farm density on deformed map with farms included in clusters and (b) corresponding farms on the original map including the main cities located near the significant clusters

to 1999,1998 to 2000$), 0.56$ at the $3-\mathrm{yr}$ lag, and 0.47 at the 4-yr lag.

The $95 \%$ quantile interval of the Hellinger distance under $\mathrm{H}_{0}$ calculated after temporal permutations of ASCS residuals were 0.1392 to 0.1515 . The global Hellinger distance values were beyond these bounds for $1996(\mathrm{Hel}=0.1563)$ and $2000(\mathrm{Hel}=0.1648)$, pointing out that those years were spatially different from the others.

\section{DISCUSSION}

In addition to the Gaussian behavior, the logarithmic scaling of SCC shows better correlation than other SCC indicators with variables of importance to farmers (milk yield loss, milk casein percentage, and probability of positive bacterial culture in the milk; IDF, 1997). Consequently, SCS can be used both to study variation factors for SCC (Barnouin et al., 2004) and for the genetic evaluation of bulls and cows to improve resistance to mastitis (Shook and Schutz, 1994). The SCS is mainly used as a continuous variable, because no consensus exists for the choice of a pertinent economic or biological threshold value (IDF, 1997). Moreover, SCS as a continuous variable is still considered to be the best SCC indicator for studies summarizing somatic cell levels at the national or regional level (IDF, 1997).

Besides surveys using a representative set group of herds at the national level, which can highlight the main factors associated with SCS, the identification of SCS spatial clusters could display focused variation factors (e.g., climate accidents, emerging strains or pathogens, or wide local use of deleterious bulls) that are too infrequent, with respect to the general population, to be cleared up through large-scale spatial studies. The detection of SCS spatiotemporal clusters could help local DHIA technicians supervise mastitis risk by detecting areas with improved or worsened udder health. Controls and interventions could be spatially and temporally targeted, from focused epidemiological studies to the follow-up of management or environmental conditions.

The results of the linear regression models were consistent with previously published results indicating significant effects of breed (Barnouin et al., 1999), parity (Laevens et al., 1997), and calving season (Barnouin et al., 2004) on ASCS. The decrease in ASCS with increasing altitude could be explained by the high dairy specialization of farms located in mountainous areas, because dairy specialization is associated with low ASCS in France (Barnouin et al., 2004). Nevertheless, some of the most significant known risk factors for ASCS were not available in the present work, which is consistent with the low $\mathrm{R}^{2}$ of the models. In particular, housing, milking, and climatic conditions, which are recognized variation factors, were not included in the model. Consequently, the clusters detected could be linked to some missing factors. Because the aim of the study was to propose a generic method to detect ASCS clusters, the factors included in the models must be considered mainly illustrative. Complementary risk and protection factors could easily be included in the 


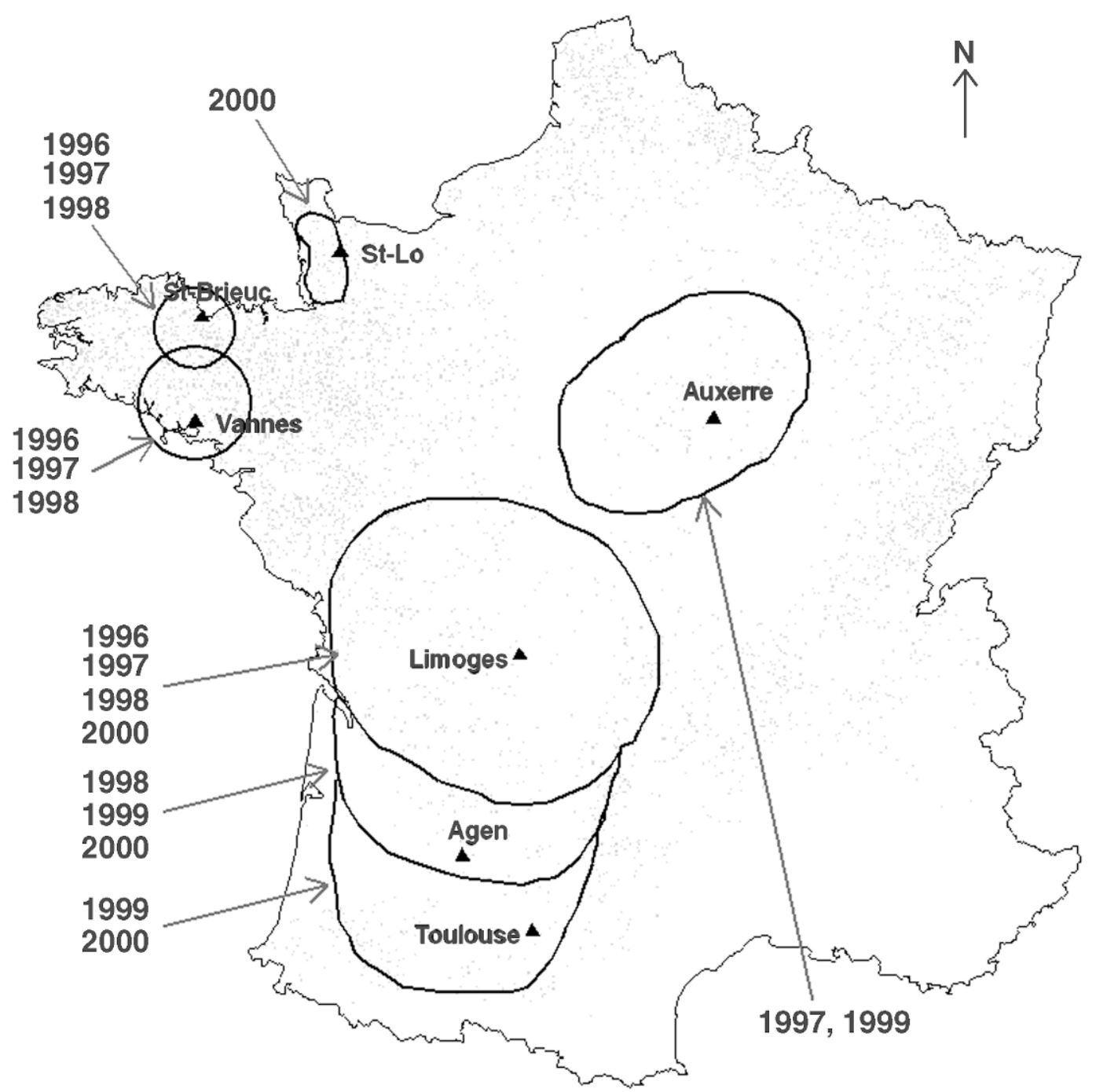

Figure 6. Clusters of annual SCS detected in France during the study period (1996 to 2000) with indication of the detection years.

models if available later, and a new cluster detection could be performed.

During the study period, several clusters were detected. Not all were perceptible from the maps of ASCS intensity, which showed only rough trends and appeared sensitive to isolated high values. Furthermore, we noticed some differences between areas detected using raw data and those using ASCS residuals.
Among these differences, 3 could be linked to the breed. Indeed, the area around St-Lo had numerous herds with Normande cows, and the Normande breed has higher ASCS than the Holstein or Montbéliarde. Consequently, if cluster detection is not adjusted for breed, Normande herds could be detected more frequently as parts of high ASCS clusters. By contrast, the Montbéliarde, which is the main breed around Ves-

Table 4. Global Hellinger distance and quantile interval of the randomization distribution for the analysis of annual SCS residuals (dairy herds in France, $\mathrm{n}=5,210,1996$ to 2000)

\begin{tabular}{llllll}
\hline & 1996 & 1997 & 1998 & 1999 & 0.1516 \\
\hline $\begin{array}{l}\text { Global Hellinger distance } \\
\begin{array}{l}\text { Quantile interval of the } \\
\text { randomization distribution } \\
\text { (bilateral risk of } 2.5 \%)\end{array}\end{array}$ & 0.1563 & 0.1505 & 0.1503 & 0.1648 \\
\hline
\end{tabular}




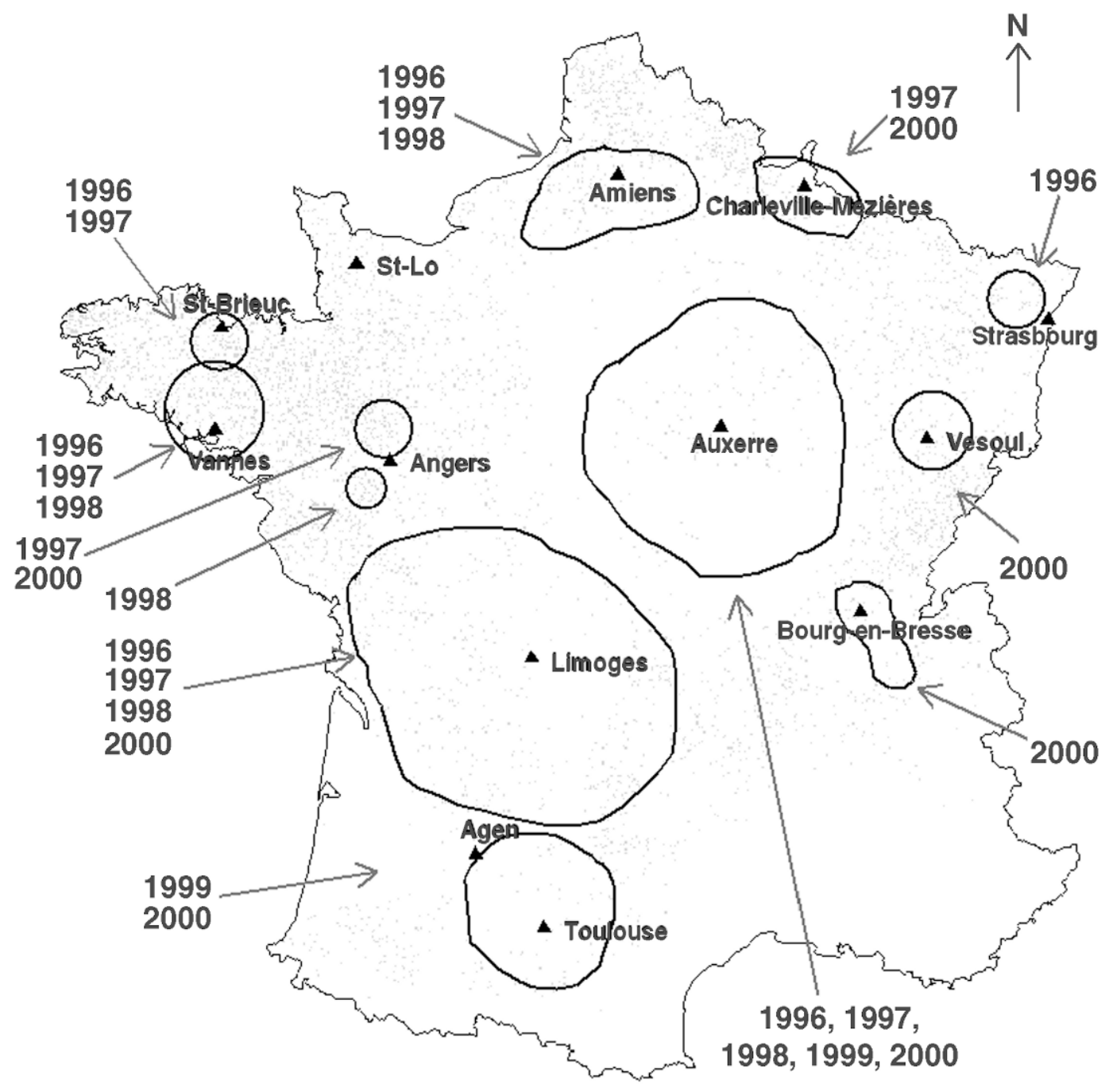

Figure 7. Clusters of annual SCS residuals detected in France during the study period (1996 to 2000) with indication of the detection years.

oul and Bourg-en-Bresse, has lower ASCS than the Holstein. Consequently, potential clusters that include high numbers of Montbéliarde herds are hidden if the detection is not adjusted for breed. Performing a regression model before the cluster detection would be a recommended procedure to focus on unexplained clustering (Klassen et al., 2005) and to try to identify new risk factors that are relevant on broader spatial scales.

Among the clusters detected on ASCS residuals, 4 were focused on small areas in the northwest with high farm density, whereas 5 were related to areas in the north and east with medium farm densities. The last 3 clusters, which were more spatially diffuse, corresponded to areas with low farm density located in the southwest, the west-central area, and the northcentral area. Some clusters were detected over several years, which was consistent with the strong correlation of ASCS residuals between years. The 2 clusters persistent over the 5-yr period were located around Auxerre in the north-central area and in the southwest. This southwestern cluster progressively spread to the south during the period studied. This result was consistent with the numerous successive increases in ASCS highlighted in the southwest. By contrast, we observed a high number of successive ASCS decreases in the northwest, a trend that could explain why the clusters around St-Brieuc and Vannes were not detected after 1998. Over the whole period studied, 2 trends were noticed: a disappearance of clusters in the northwest, and a cluster extension from the westcentral area to the southwest. Such a statement could explain the spatial discrepancy that was highlighted in 1996 and 2000 by temporal permutation tests: 1996 
could be the beginning of an improvement in the northwest, whereas 2000 would be the beginning of a deterioration in the southwest. Moreover, the discrepancy of the year 2000 was emphasized by the appearance of 2 new clusters in the east-central region.

The general trend of a decrease in ASCS can be attributed to the implementation by DHIA of preventive measures, milking visits, and intervention programs to improve dairy management and mastitis control. The northwest, which is the main dairy basin in France (50\% of the farms) and which is more technically advanced, was associated less with clusters of high ASCS values (except around Vannes). Conversely, the areas with several clusters detected were not specialized in dairy production, and mainly focused on bovine and ovine meat and on cereal production. To explain the factors associated with the clusters more precisely, a local analysis would be necessary. An examination of relevant factors to explain most of the clusters detected is presently out of reach. Only local DHIA technicians and mastitis experts have the relevant information on local events, decisions, or singularities that could locally influence ASCS. For instance, the veterinarian working as a mastitis expert for the DHIA in the Morbihan department (region of Vannes) proposed a hypothesis to explain the Vannes cluster. She argued that in the $1990 \mathrm{~s}$, a significant number of farms in the department widely used a deleterious bull through artificial insemination, which produced mastitis-susceptible cows. Moreover, the bovine spongiform encephalopathy crisis led to fewer animal replacements in the Morbihan department, a practice that meant cows with high SCS were kept in the herds longer than usual.

The cluster detection method we proposed can take into account the variation factors of the outcome variable and the heterogeneity of the background population. This approach can be applied on both discrete and quantitative data, and is able to detect clusters in areas of low farm density. Another advantage of the method is that it is not necessary to have a priori precision of either the number or the position of spatial clusters.

\section{CONCLUSIONS}

The cluster detection method based on Hellinger distance was a useful tool for detecting spatial clusters of SCS. Identification of regional differences for the disease could help the local DHIA manage mastitis risk more accurately.

The method can also be used to detect clusters of low values, which could be useful to identify regions and areas with a very good control of ASCS. Moreover, the generalized detection of spatiotemporal patterns for milk production and hygiene parameters could be an efficient way to dynamically improve milk quality at the national level.

\section{REFERENCES}

Ahrens, C., N. Altman, G. Casella, M. Eaton, J. T. G. Hwang, J. Staudenmayer, and C. Stefanescu. 2001. Leukemia clusters in update New York: How adding covariates changes the story. Environmetrics 12:659-672.

Ali, A. K. A., and G. E. Shook. 1980. An optimum transformation for somatic cell concentration in milk. J. Dairy Sci. 63:487-490.

Barkema, H. W., H. A. Deluyker, Y. H. Schukken, and T. J. G. M. Lam. 1999. Quarter-milk somatic cell count at calving and at the first six milkings after calving. Prev. Vet. Med. 38:1-9.

Barkema, H. W., Y. H. Schukken, T. J. G. M. Lam, M. L. Beiboer, G. Benedictus, and A. Brand. 1998. Management practices associated with low, medium, and high somatic cell counts in bulk milk. J. Dairy Sci. 81:1917-1927.

Barnouin, J., M. Chassagne, S. Bazin, and D. Boichard. 2004. Management practices from questionnaire surveys in herds with very low somatic cell score through a national mastitis program in France. J. Dairy Sci. 87:3989-3999.

Barnouin, J., N. Geromegnace, M. Chassagne, N. Dorr, and P. Sabatier. 1999. Facteurs structurels de variation des niveaux de comptage cellulaire du lait et de fréquence des mammites cliniques dans 560 élevages bovins répartis dans 21 départements français. Anim. Prod. 12:39-48.

Busato, A., P. Trachsel, M. Schallibaum, and J. W. Blum. 2000. Udder health and risk factors for subclinical mastitis in organic dairy farms in Switzerland. Prev. Vet. Med. 44:205-220.

Carpenter, T. E. 2001. Methods to investigate spatial and temporal clustering in veterinary epidemiology. Prev. Vet. Med. 48:303-320.

Draper, N. R., and H. Smith. 1996. Applied Regression Analysis. John Wiley \& Sons, New York, NY

Elliott, P., and J. Wakefield. 2001. Disease clusters: Should they be investigated, and, if so, when and how? J. Roy. Statist. Soc. A 164:3-12.

Ely, L. O., J. W. Smith, and G. H. Oleggini. 2003. Regional production differences. J. Dairy Sci. 86:E28-E34.

Gibbs, A. L., and F. E. Su. 2002. On choosing and bounding probability metrics. Int. Stat. Rev. 70:419-435.

Harmon, R. J. 1994. Physiology of mastitis and factors affecting somatic cell counts. J. Dairy Sci. 77:2103-2112.

Huffman, E. M., R. Mortimer, J. D. Olson, L. Ball, and P. W. Farin. 1984. Risk factors for prebreeding pyometra on four Colorado dairy farms. Prev. Vet. Med. 2:785-790.

IDF. 1997. Recommendations for Presentation of Mastitis-Related Data. Bulletin no. 321. International Dairy Federation, Brussels, Belgium.

Klassen, A. C., M. Kulldorff, and F. Curriero. 2005. Geographical clustering of prostate cancer grade and stage at diagnosis, before and after adjustment for risk factors. Int. J. Health Geogr. 4:1.

Laevens, H., H. Deluyker, Y. H. Schukken, L. De Meulemeester, R. Vandermeersch, E. De Muelenaere, and A. De Kruif. 1997. Influence of parity and stage of lactation on the somatic cell count in bacteriologically negative dairy cows. J. Dairy Sci. 80:3219-3226.

Manly, B. F. J. 1991. Randomization and Monte Carlo Methods in Biology. Chapman \& Hall, London, UK.

Marshall, R. J. 1991. A review of methods for the statistical analysis of spatial patterns of disease. J. Roy. Statist. Soc. A. 154:421441

Norstrom, M., D. U. Pfeiffer, and J. Jarp. 1999. A space-time cluster investigation of an outbreak of acute respiratory disease in Norwegian cattle herds. Prev. Vet. Med. 47:107-119. 
Oleggini, G. H., L. O. Ely, and J. W. Smith. 2001. Effect of region and herd size on dairy herd performance parameters. J. Dairy Sci. 84:1044-1050.

Perez, A. M., M. P. Ward, P. Torres, and V. Ritacco. 2002. Use of spatial statistics and monitoring data to identify clustering of bovine tuberculosis in Argentina. Prev. Vet. Med. 56:63-74.

R Development Core Team. 2004. R: A Language and Environment for Statistical Computing. R Foundation for Statistical Computing, Vienna, Austria.

Schmidt-Madsen, P. 1975. Fluoro-opto-electronic cell-counting on milk. J. Dairy Res. 42:227-239.

Senoussi, R., J. Chadoeuf, and D. Allard. 2000. Weak homogenization of points processes by space deformations. Adv. Appl. Probl. 32:948-959.

Shook, G. E., and M. M. Schutz. 1994. Selection on somatic-cell score to improve resistance to mastitis in the United States. J. Dairy Sci. 77:648-658.
Silverman, B. W. 1986. The kernel method for univariate data. Pages 34-94 in Density Estimation for Statistics and Data Analysis. D. Cox, D. Hinkley, D. Rubin, and B. Silverman, ed. Chapman \& Hall, London, UK.

Timander, L. M., and S. McLafferty. 1998. Breast cancer in West Islip, NY: A spatial clustering analysis with covariates. Soc. Sci. Med. 46:1623-1635.

Waller, L. A. 2000. A civil action and statistical assessments of the spatial pattern of disease: Do we have a cluster? Regul. Toxicol. Pharmacol. 32:174-183.

Ward, M. P., and T. E. Carpenter. 2000. Techniques for analysis of disease clustering in space and in time in veterinary epidemiology. Prev. Vet. Med. 45:257-284.

Wartenberg, D. 2001. Investigating disease clusters: why, when and how? J. Roy. Statist. Soc. A. 164:13-22. 